\title{
NEW OSTROWSKI LIKE INEQUALITIES INVOLVING THE FUNCTIONS HAVING HARMONIC $h$-CONVEXITY PROPERTY AND APPLICATIONS
}

\section{Bandar Bin-Mohsin, Muhammad Uzair Awan, Muhammad Aslam Noor, Marcela V. Mihai and Khalida InAYat NoOR}

\begin{abstract}
Some new Ostrowski type inequalities are established for the class of harmonic $h$ convex functions. Several new and known special cases, which can be derived from our main results, are also discussed. Applications to special means of some of our main results are also discussed. Results obtained in this paper continue to hold for these special cases. Techniques of this paper may lead to further research in this dynamic field.
\end{abstract}

Mathematics subject classification (2010): 26D15, 26A51.

Keywords and phrases: Convex functions, harmonic, functions, Ostrowski inequality.

\section{REFERENCES}

[1] M. A. Ardic, A. O. Akdemir, E. Set, New Ostrowski like inequalities for GG-convex and GAconvex functions, Math. Ineq. Appl., 19 (4) (2016), 1159-1168.

[2] F. Al-Azemi, O. CAlin, Asian options with harmonic average, Appl. Math. Inf. Sci. 9(6), 1-9 (2015).

[3] W. W. BRECKNER, Stetigkeitsaussagen fiir eine Klasse verallgemeinerter konvexer funktionen in topologischen linearen Raumen, Pupl. Inst. Math. 23, 13-20, (1978).

[4] G. CRistescu And L. Lups A, Non-connected Convexities and Applications, Kluwer Academic Publishers, Dordrecht, Holland, 2002.

[5] G. Cristescu, M. A. Noor, M. U. Awan, Bounds of the second degree cumulative frontier gaps of functions with generalized convexity, Carpathian J. Math. 31(2), 173-180, (2015).

[6] S. S. DRAGOMIR, Inequalities of Hermite-Hadamard type for h-convex functions on linear spaces, RGMIA Res. Rep. Coll. Article 72, (2013).

[7] S. S. Dragomir, J. PeČArić, L. E. Persson, Some inequalities of Hadamard type, Soochow J. Math. 21, 335-341, (1995).

[8] S. S. Dragomir, T. M. Rassias, Ostrowski type inequalities and applications in numerical integration, Springer Netherlands, (2002).

[9] E. K. Godunova, V. I. Levin, Neravenstva dlja funkcii sirokogo klassa, soderzascego vypuklye, monotonnye i nekotorye drugie vidy funkii, Vycislitel. Mat. i. Fiz. Mezvuzov. Sb. Nauc. Trudov, MGPI, Moskva. (1985) 138-142, (in Russian).

[10] I. IsCAN, Ostrowski type inequalities for harmonically s-convex functions, Konuralp J. Math., 3(1), 63-74, (2015).

[11] I. IsCAN, Hermite-Hadamard type inequalities for harmonically convex functions, Hacettepe J. Math. Stat. 43(6), 935-942, (2014).

[12] A. Kilbas , H. M. SRivastaVA, J. J. Trujillo, Theory and applications of fractional differential equations, Elsevier B.V., Amsterdam, Netherlands, (2006).

[13] M. V. Mihai, M. A. Noor, K. I. Noor, M. U. Awan, Some integral inequalities for harmonic h-convex functions involving hypergeometric functions, Appl. Math. Comput. 252, 257-262, (2015).

[14] C. P. Niculescu, L. E. Persson, Convex functions and their applications, A contemporary approach, CMC Books in Mathematics, Springer (New York, 2006).

[15] M. A. NOoR, K. I. Noor, M. U. AwAN, Integral inequalities for coordinated harmonically convex functions, Complex Var. Elliptic Equat. DOI:10.1080/17476933.2014.976814, (2014). 
[16] M. A. Noor, K. I. Noor, M. U. AwAn, S. Costache, Some integral inequalities for harmonically h-convex functions, U. P. B. Sci. Bull., Series A. 77(1), 5-16, (2015).

[17] A. OstrowsKI, Uber die Absolutabweichung einer differentienbaren Funktionen von ihren Integralmittelwert, Comment. Math. Hel. 10, 226-227, (1938).

[18] J. PARK, Hermite-Hadamard-like and Simpson-like type inequalities for harmonically convex functions, Int. J. Math. Anal. 8(27), 1321-1337, (2014).

[19] H.-N. ShI AND J. ZHANG, Some new judgement theorems of Schur geometric and Schur harmonic convexities for a class of symmetric functions, J. Inequal. Appl. 2013(2013), 527.

[20] S. VArošAnEC, On h-convexity, J. Math. Anal. Appl. 326, 303-311, (2007). 